\title{
Prospects of Molecular Biotechnology in Diagnostics: Step Towards Precision Medicine
}

\author{
Praveen Sharma $^{1} \cdot$ Shailendra Dwivedi $^{1}$
}

Published online: 3 April 2017

(C) Association of Clinical Biochemists of India 2017

Molecular diagnostic tools as nucleotide sequences have been extensively utilized through persistent advancement in the molecular biology and biotechnology in diagnosis of cancerous, neurological, genetic and microbial diseases. Automated and more cutting-edge technology have revolutionized the amplification and screening of nucleic acid sequence for microbial agents, using various nucleic acid amplification technologies such as PCR, real time PCR etc. These robotic systems have provided perfection of not only in assay efficiency but also in quality control of the tests and have contributed to the standardization of traditional biomarkers. It is recognised since Gregor Mendel that factors are accountable for development of characters and after the draft of human genome the depiction of genotype to phenotype relationship appears more bright and diverse. These functions of genes and proteins have been deliberated by post-genomics cutting-edge technologies such as expression profiling using DNA microarray, proteomics, and single nucleotide polymorphisms analysis, coupled with bioinformatics. Now, genetics has become the driving force in medical research and is now prepared for integration into medical practice. Human genome draft (bio-informatics) with innovation in current techniques now opens new vistas in the fields of novel therapeutics such as Pharmacogenomics, Chemo-informatics and Nutrigenomics that may revolutionize the management of untreated disease and disorders.

The recent innovation of molecular technologies together with a multidisciplinary interaction of several fields led to the expansion of genomics, which focusses on the detection

Praveen Sharma

praveensharma55@gmail.com

1 Department of Biochemistry, All India Institute of Medical Sciences, Jodhpur 342005, India of pathogenic events at the genome level. The structural and functional genomics tactics have now pinpointed the technical challenge in the screening of disease-related genes and the recognition of their structural variations or elucidation of gene function. Several promising technologies and diagnostic applications of structural genomics are now preparing a large database of disease-genes, genetic alterations etc. by mutation scanning and DNA chip technology. Further the functional genomics also exploring the expression genetics (hybridization-, PCR- and sequence-based technologies), two-hybrid technology, next generation sequencing with Bioinformatics and computational biology. Progresses in microarray "chip" technology as microarrays have permitted the parallel analysis of gene expression patterns of thousands of genes concurrently. Sequence information poised from the genomes of several individuals is leading to the prompt discovery of single nucleotide polymorphisms or SNPs. Further, evolutions of genetic engineering have also revolutionized immunoassay biotechnology via engineering of antibody-encoding genes and the phage display technology. The Biotechnology shows an important role in the development of diagnostic assays in response to an outburst or critical disease response need. An example is the field validation of the SNP assay for Equine Herpesvirus-1 during an outburst on 3 California race tracks in 2006/2007, and lately the development and validation of a real-time PCR assay for the detection of very virulent variant of Infectious Bursal Disease virus in 2009 [1].

\section{Molecular Diagnosis: Present Status}

The central categories of diagnostics are clinical chemistry, immunology, haematology, microbiology and molecular diagnostics. The diagnostics industry continues to invent in 
all of these significant areas, and molecular diagnostics has confined particular devotion in recent years because of the deep insights, these types of tests bring to diagnosis and treatment. The recognition and fine characterization of the genetic basis of the disease in query is vital for precise provision of diagnosis. Gene discovery offers invaluable insights into the mechanisms of disease, and gene-based markers that permit clinicians not only to evaluate disease predisposition but also to design and implement improved diagnostic methods. The latter is of great significance, as the enormous and variety of molecular defects demands the utilization of multiple rather than a single mutation detection platform. Molecular diagnostics is now a clinical reality with its roots deep into basic study of gene expression and function. The technique is utilized to diagnose and screen disease, notice risk, and choose which therapies will work best for individual patients. By examining the specifics of the patient and their disease, molecular diagnostics endorses the prospect of personalised medicine. These tests are fruitful in various medical specialism, including infectious disease, oncology, human leukocyte antigen typing, coagulation, and pharmacogenomics i.e. genetic prediction of which drugs will outfit best.

The exploitation of high throughput genotyping tools for the characterization and screening of single nucleotide polymorphisms (SNPs) eventually can lead to the determination of the exclusive molecular signature of an individual in a relatively short period of time and it can predict the susceptibility of any diseases. As The double mutation, A to $\mathrm{T}$ transversion at 1762 and $\mathrm{G}$ to $\mathrm{A}$ transition at 1764 , is often present in patients with chronic hepatitis, hepatocellular carcinoma, and hepatitis and less often in asymptomatic carriers, in immunosuppressed patients, and in carriers without HBV markers. Thus individual drug responses can be forecasted from known genetic variances correlated with a drug effect. Drug response of Maraviroc efficacy depends on CCR5 alleles in HIV-1 infections and Interferon-alpha drug efficacy also decided by the alleles of IL28B in Hepatitis C infections. Thus, this will permit the physician to decide the patient with a selective drug treatment.

The utilization of targeted therapies requires the identification of specific targets that play a key role in cancer cell growth and survival. The expression analysis of human epidermal growth factor receptor 2 protein (HER-2), which is expressed at high levels on the surface of some cancer cells, becomes good target of therapies, including trastuzumab $\left(\right.$ Herceptin $\left.^{\circledR}\right)$, which is permitted to treat certain breast and stomach cancers that overexpress HER-2. Further characterization of mutant (altered) proteins that drive cancer progression is also now utilized in targeted therapies. For example, the cell growth signalling protein BRAF is present in an altered form (known as BRAF V600E) in many melanomas. Vemurafenib (Zelboraf ${ }^{\circledR}$ ) targets this mutant form of the BRAF protein and is approved to treat patients with inoperable or metastatic melanoma that contains this altered BRAF protein.

Researchers also exploring abnormalities in chromosomes that are exist in cancer cells but not in normal cells. Often these chromosome abnormalities result in the creation of a fusion gene (a gene that incorporates parts of two different genes) whose product, called a fusion protein, may initiate cancer development and progression. Such fusion proteins are probable targets for targeted cancer therapies. For example, imatinib mesylate $\left(\right.$ Gleevec $^{\circledR}$ ) targets the BCR-ABL fusion protein that is generated from pieces of two genes that get joined together in some leukemia cells and promotes the growth of leukemic cells [2].

Few studies have proven the sulforaphane, butyrate, and allyl sulfur are active inhibitors of histone deacetylase (HDAC). HDAC inhibition was associated with global increases in histone acetylation, enhanced interactions of acetylated histones with the promoter regions of the P21 and BAX genes, and higher expression of p21Cip1/Waf1 and BAX proteins. Notably, sulforaphane has been described to decrease HDAC activity in humans. Upcoming research likely desires to relate HDAC changes in humans to a change in a cancer-related process. Furthermore, since acetylation is only one process to regulate histone homeostasis. Cancer pathway based signalling studies have perceived up regulation of apoptosis-related genes such as PIK3C3 and Akt1. PIK3C3 belongs to the phosphoinositide (PI) 3-kinase family involved in both receptor-mediated signal transduction and intracellular trafficking. PI3 K proteins produce specific inositol lipids involved in the regulation of cell growth, proliferation, survival, differentiation, and cytoskeletal changes. Additionally, researchers have shown up regulation of MAPK related genes such as heat shock protein HSPA1B, the fibroblast growth factor FGF2, and FOS, a major component of the activator protein-1 (AP-1) transcription factor complex, which includes members of the JUN family. The AP-1 transcription factor is a regulator of processes essential for normal growth and development as well as carcinogenesis. Similarly, pro-inflammatory and anti-inflammatory interleukin's genotypes and gene expression have established their role in survival of prostate cancer patients [3-5]. Thus molecular diagnostics is going to swing the whole paradigm of screening and treatment. Presently, a great number of blood, hair, semen, and tissue samples are screened and characterized annually worldwide in both public and private laboratories, and the number of genetic tests offered is continuously increased year by year. So the population and individual predisposed 
to any disease and disorders will be not only diagnosed earlier but also treatment can be planned at pre-natal stage.

\section{Personalized Medicine: An Integration of Diagnostics with Therapeutics}

In a large patient sample size, a medicine and drug that is advantageous in many patients often fails to act in some other patients. Moreover, when it does work, it may cause adverse side effects, even death, in a small number of patients. Though large individual variability in drug efficacy and safety has been recognised to exist since the establishment of human medicine but the cause was beyond to knowledge. On the other hand, the request to overcome such variation has received more consideration now after the draft of human genome than ever before. The human genome sequence provides an exclusive database of human evolution that varies among populations and individuals. Sequence differences in drug target proteins, drugmetabolizing enzymes, and drug transporters can change drug efficacy, drug side effects, or both to cause variable drug responses in individual patients. From this prospect, the availability of the complete human genome sequence has made it possible to analyse the impact of variations of the human genome sequence on the pathogenesis of important diseases and the response to drug therapy at an expedite rate in recent years. The fast accumulation of information on genome-disease and genome-drug interactions has also prompted the transformation of pharmacogenetics into a new entity of human genetics, pharmacogenomics and, at the same time, provided a rationale for the hope that individualized medicine can be accomplished in the near future.

Few pharmaceutical companies and research development agencies are developing a precise haplotyping scheme to identify individuals/patients who will be benefiting from which type of drug in a particular disease and disorders. Further, Nutrigenomics is also sprouting and encouraging a better management of patients. Several bioactive food components, including both essential and nonessential nutrients, can regulate gene expression patterns. Thus, nutrigenomics is providing the effects of ingested nutrients and other food components on gene expression and gene regulation, i.e. diet-gene interaction in order to spot the dietetic components having beneficial or detrimental health effects. Nutritional genomics (nutrigenomics), the junction between health, diet, and genomics, is inclined on epigenetic, transcriptomics, and proteomics courses of biology. Thus, it will assist in determining the individual nutritional requirements based on the genetic makeup of the person (personalized diet) as well as the association between diet and chronic diseases like cancer, opening new vistas to understanding the complexity of various diseases [6].

In the future, molecular diagnostics will persist to be of critical prominence to public health globally as it provides physicians with critical information based on the early detection of pathogens and subtle changes in patients' genes and chromosomes, allowing for earlier diagnosis, selection of appropriate therapies and monitoring of disease progression. However, there are major obstacles to overcome before the execution of these tests in clinical laboratories, such as which to employ, the choice of technology and equipment, and issues such as cost-effectiveness, accuracy, reproducibility. Presently PCR-based testing outweighs; however, alternative technologies aimed to discover genome complexity without PCR are anticipated to gain momentum in the coming years as sequencing devices are more expensive at present. Also, advances of integrated silicon chips mounted with biomolecules is now going to revolutionize the concept of traditional wet lab to "lab-on-a-chip". Thus it would be conceivable to analyse thousands of genes/proteins in hours from low amount/ single cell sample. Thus the coming era of molecular biotechnology will be innovatory, that would change diagnostics, treatment and therapy.

\section{References}

1. Lianidou E, Ahmad-Nejad P, Ferreira-Gonzalez A, Izuhara K, Cremonesi L, Schroeder ME, et al. Advancing the education in molecular diagnostics: the IFCC-initiative "clinical molecular biology curriculum" (C-CMBC); a ten-year experience. Clin Chim Acta. 2014;436:5-8.

2. Nishi A, Milner DA Jr, Giovannucci EL, Nishihara R, Tan AS, Kawachi I, Ogino S, et al. Integration of molecular pathology, epidemiology and social science for global precision medicine. Expert Rev Mol Diagn. 2016;16:11-23.

3. Dwivedi S, Shukla KK, Gupta G, Sharma P. Non-invasive biomarker in prostate carcinoma: a novel approach. Ind J Clin Biochem. 2013;28:107-9.

4. Dwivedi S, Goel A, Mandhani A, Khattri S, Sharma P, Misra S, et al. Functional genetic variability at promoters of pro-(IL-18) and anti-(IL-10) inflammatory affects their mRNA expression and survival in prostate carcinoma patients: five year follow-up study. Prostate. 2015;75:1737-46.

5. Dwivedi S, Goel A, Khattri S, Mandhani A, Sharma P, Misra S, et al. Genetic variability at promoters of IL-18 (pro-) and IL-10 (anti-) inflammatory gene affects susceptibility and their circulating serum levels: an explorative study of prostate cancer patients in North Indian populations. Cytokine. 2015;74:117-22.

6. Dwivedi S, Goel A, Khattri S, Mandhani A, Sharma P, Pant KK. Tobacco exposure by various modes may alter pro-inflammatory (IL-12) and anti-inflammatory (IL-10) levels and affects the survival of prostate carcinoma patients: an explorative study in North Indian population. Biomed Res Int. 2014. doi:10.1155/2014/ 158530 . 